\title{
Multi-Classifier Framework for Medical Image Analysis using Mutual Information Criterion
}

\author{
Edbert Rajan.R \\ Research Scholar \\ Singania University \\ Rajasthan
}

\author{
Prasadh.K, PhD. \\ Mookambika Technical Campus \\ Mannathur \\ Kerela
}

\begin{abstract}
The efficacy of the medical image analysis with the level-set shape along with fractal texture and intensity features to discriminate PF (Posteriorfossa) tumor from other tissues in the brain image. Further, extracted features may not be adequate to differentiate amongst the medical images. To enhance the medical image processing, to devise an automotive subjective optimality model for segmentation of images based on different sets of selected features from the unsupervised learning model of extracted features. After segmentation, it is necessary to classify the image based on different classes it belongs to. To classify a medical image, a multiple classifier framework and classify the image based on the classes like normal body cells, infected cells, and highly infected cells. The classifier is designed based on the mutual information coefficient of the selected features underwent for image segmentation procedures. The classification is done with set of rotation invariant features being selected on the lines of subjective-optimality and different classifiers are organized using different features sets trained in different data. An experimental performance is evaluated with benchmark data sets extracted from research repositories of both real and synthetic data sets. The performance parameter used for the analysis of the proposed multi-classifier framework using mutual concept criterion [MFMCC] for medical image analysis are Multiple Class intensity, Mutual information coefficient of rich features and efficiency.
\end{abstract}

\section{Keywords}

Mutual information, Feature selection, Medical image analysis, Feature extraction, Scale Invariant Feature Extraction.

\section{INTRODUCTION}

Over the previous decades, medical imaging has turn out to be customary for steering patient analysis care. The abilities of medical imaging devices to scrutinize practical and anatomical information of the interior formations of the patient and to symbolize it by images have completed these images critical in medical image diagnosis. At present diverse medical imaging modalities are attained from the medical scanning devices.

Feature collection is classically a search crisis for discovering a best possible or Sub-optimal division of $\mathrm{m}$ features not in unusual features. Feature collection is significant in numerous pattern detection troubles for not including immaterial and redundant features. It permits reducing system difficulty and giving out time and frequently develops the detection correctness. Numerous feature valuation functions have been employed mainly functions that determine distance, information, dependence, and steadiness. The segmentation of textured images is an extensive reputation crisis in processor visualization, which has been addressed from different viewpoints, with difference models, and MRFs, being the most widespread approaches. As image strength is a deprived prompt for texture segmentation, altering the image by a Gabor filter bank is usually employed as a feature-extraction preprocessing pace which consequences in noticing texture information that exists in at diverse frequency channels. Region based segmentation algorithms which consequently set pixels into regions consistent with the closeness of the filter retorts at these points are more worldwide and consequently more efficient, opposing to edge based algorithms which effect in bogus edges, owing to the intrinsically arbitrary nature of textures.

Numerous classifiers are positions of classifiers whose character forecasts are shared in various ways to categorize novel shared predictions are instances figure 1. Different names group methods for manifold classifiers are board, classifier synthesis, mixture, aggregation. Integration should progress prognostic accurateness. An essential state for the approach to be constructive is that member classifiers should contain an extensive level of discrepancy, i.e., they construct error separately with value to one another.

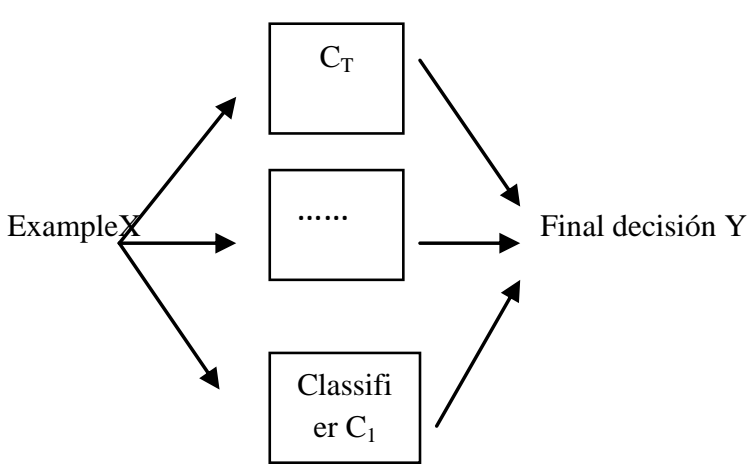

Figure 1: Múltiple classifiers

Two classifiers are varied, if they create diverse errors on a novel object. Imagine a set of three classifiers $\left\{\mathrm{h}_{1}, \mathrm{~h}_{2}, \mathrm{~h}_{3}\right\}$ and a novel object $\mathrm{x}$.

- If all are the same, then when $\mathrm{h}_{1}(\mathrm{x})$ is wrong, $\mathrm{h}_{2}(\mathrm{x})$ and $\mathrm{h}_{3}$ (x) will be also wrong

- If the classifier faults are uncorrelated, then when $\mathrm{h}_{1}(\mathrm{x})$ is wrong, $h_{2}(x)$ and $h_{3}(x)$ might be correct. Diverse approaches are employed to make numerous systems. They are, 


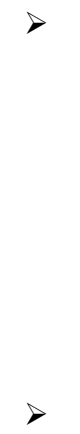
over expanded data sets

Homogeneous classifiers algorithm

- Bagging (Breiman)

- Boosting (Freund, Schapire)

- Multiple partitioned data

- Multi-class specialized systems, (e.g. ECOC pair wise classification)

\section{Heterogeneous classifiers algorithms over the same data \\ - Voting or rule-fixed aggregation \\ - Stacked simplification or meta-learning}

The principle of this study is to discover an information theoretic approach to the feature collection crisis for CAD applications. The approach uses the common information MI idea as the feature collection principle. By the way, MI actions the information content of a specified feature relating to the resolution task at hand. Hypothetically, the MI measure presents three major rewards over other techniques. First the MI deals with the common statistical reliance among variables, different to the linear correlation co-efficient. Second, the MI is invariant to monotonic alterations executed on the variables, different to linear measurement reducers' for instance principal constituent examination. At last, the MI feature collection approach is self-sufficient of the conclusion algorithm, therefore reducing computational difficulty divergent to the approach.

In this work, a multiple classifier is presented for the medical images based on the normal body cells, infected cells, and highly infected cells. The classifier is designed based on the mutual information coefficient of the selected features underwent for image segmentation procedures. The classification is done with set of rotation invariant features being selected on the lines of subjective-optimality and different classifiers are organized using different features sets trained in different data. This paper is well thought-out as follows; Section 2 deals with the review of literature. Section 3 described about the multi classifier framework for medical image analysis using mutual information criterion. Section 4 and 5 offered to Experimental evaluation with result and discussion. Finally the conclusion of this paper in Section 6.

\section{LITERATURE REVIEW}

Medical imaging is the method and procedure employed to generate images of the person body for medical purposes (medical measures looking for diagnose, or examine disease) or medicinal science (counting the revise of standard structure and physiology). Though imaging of detached organs and tissues can be achieved for medicinal reasons, such events are not regularly referred to as medicinal imaging, but quite are a division of pathology. Numerous techniques have been presented by several authors for an analysis of medical images. In [1], the author presented a straight image examination approach that employs mutual information (MI) as a metric for position. The medical image examination is vigorous and considered the amount of information common by signals. Even though it has the capability to achieve vigorous arrangement associated to

The multi-classifier framework using mutual concept criterion [MFMCC] for medical image analysis is processed under spatiotemporal image muster or object trailing in image sequences as of some optimization troubles.

Maximization of mutual information (MMI) is a similarity principle for medical image analysis. Even though its correctness and sturdiness has been established for inflexible body image segmentation, expanding MMI to non-rigid image listing is inconsequential and a vigorous field of research. The author in [2] proposed restricted mutual information (cMI) as a novel parallel appraisal for non-rigid image analysis. Using cMI structure the author includes kinetic characteristic maps derived from DCE MRI as examination conduits in MRF for tumor segmentation. The algorithm in [3] based on multi-channel MRF attains segmentation when utilizing a manual description as position accuracy.

Choosing the most instructive features from high dimensional gap is one of the recognized troubles in multispectral image categorization and prototype detection applications [4]. The regularly employed techniques for dimensionality lessening are the Principal Components Analysis (PCA) and the Linear Discriminate Analysis (LDA). The crisis can be addressed using two basic techniques: feature collection and feature mining. In [5], a feature lessening method (MI-PCA) is presented which exercises nonparametric mutual information (MI) evaluates on the components achieved using PCA. With Gabor development and maximization of MI attained state-of-theart categorization precision for image processing [6]. In [7], three diverse methodologies of band collection are accessible for hyper-spectral data sets partitioned to categorization and deterioration tasks using Information Theory actions for image analysis. The paper [8] proposed a common sequence mutual information concept to quantitatively and impartially assess the performances of disparity image feature mining techniques.

In [9], interpreting cognitive information is addressed from practical magnetic resonance (MR) images using categorization techniques. To improve the classification, the author developed a multivariate approach supported on a mutual information principle, anticipated by nearest neighbors [10]. The author in [11] proposed a technique includes k-means and improved watershed segmentation algorithm for remedial image segmentation.

However, its disadvantages cover over-segmentation and concern to forged edges. Intensity based image segmentation is finished to supervise with the disadvantages confirmed above and the assessment of k-means clustering with Gaussian distribution is discussed in [12]. In this work, we plan to classify the image based on the different types of classes by adapting the mutual information criterion used in it.

\section{MEDICAL IMAGE ANALYSIS USING MUTUAL INFORMATION CRITERION}

The proposed work is efficiently designed for performing the classification of the given medical image based on the normal body cells, infected cells, and highly infected cells. The classifier is designed based on the mutual information coefficient of the selected features underwent for image segmentation procedures. The classification is done with set of rotation invariant features being selected on the lines of subjective-optimality and different classifiers are organized d using different features sets trained in different data.

three different processes. The first process is to extract the spatial and hierarchical features using unsupervised learning 
model with SIFT approach. The second process is to segment the image based on the features. Feature selection is done using automatic subjective-optimality model. Finally, multiple classifiers are done based on the mutual information coefficient of the selected features. The architecture diagram of the proposed multi-classifier framework using mutual concept criterion is shown in figure 2 .

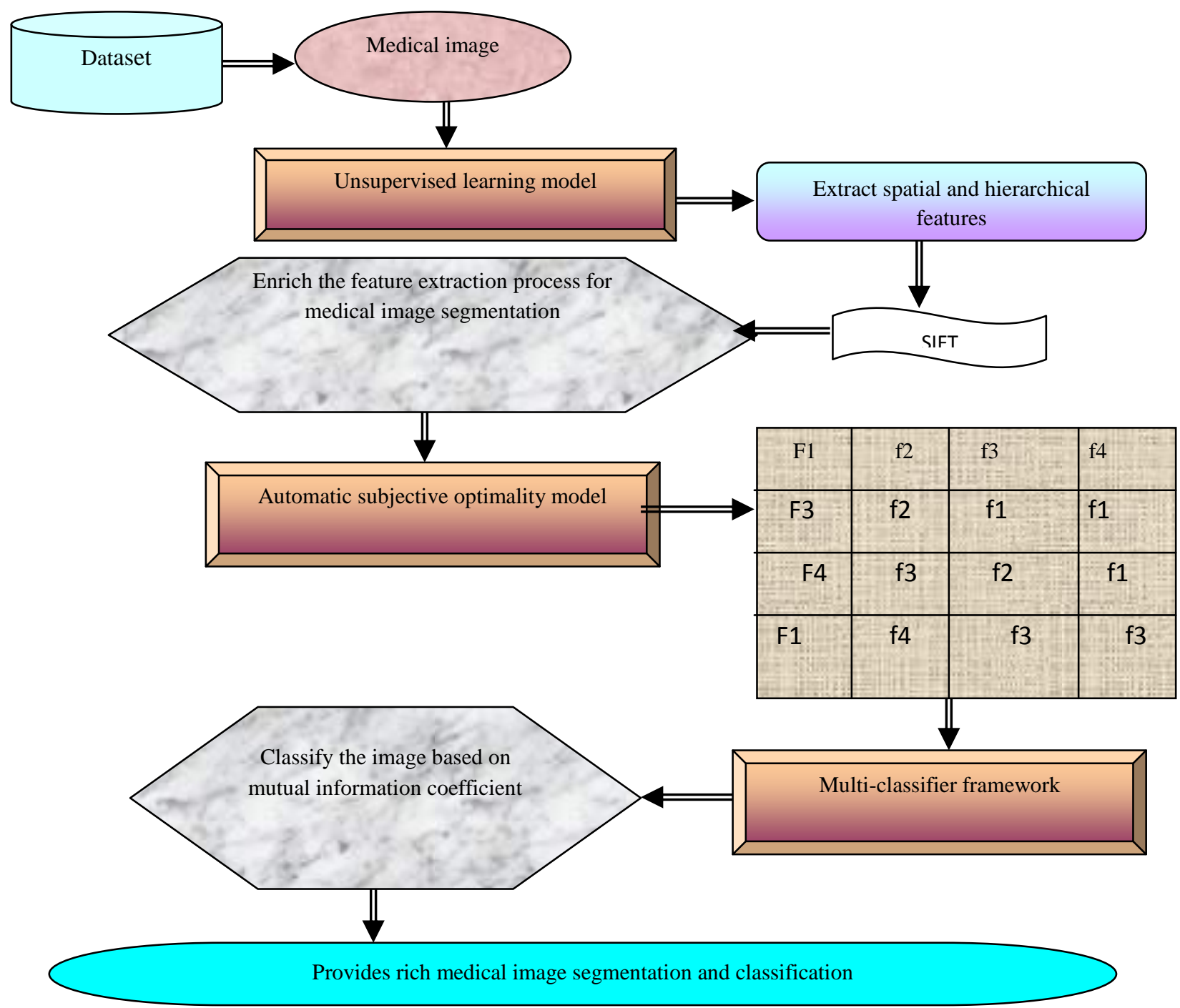

Figure 2: Architecture diagram of the proposed MFMCC

The first process employed unsupervised learning model to extract features from the medical images using spatial and hierarchical structures based on scale invariant feature transformation.

The second process devised a schematic procedure for segmentation of images based on different sets of selected features from the unsupervised learning model of extracted features. Feature selection on the medical images is done on the basis of automatic subjective-optimality model.

Finally, we plan to present a multiple classifier for the medical images based on the normal body cells, infected cells, and highly infected cells. The classifier is designed based on the mutual information coefficient of the selected features underwent for image segmentation procedures. The classification is done with set of rotation invariant features being selected on the lines of subjective-optimality and different classifiers are organized using different features sets trained in different data.

\subsection{Scale invariant feature transform for feature extraction}

By using SIFT; image has been converted into a compilation of restricted spatial and hierarchical feature vectors. Each of these features is believed to be unique and variant to any alternation, range, or alteration of the image. In the original implementation, these features can be utilized to discover individual objects in diverse images and the conversion can be unlimited to image segmentation.

Following are the major stages of computation used to extract the set of spatial and hierarchical features from medical image diagnosis: 


\subsubsection{Scale-space extrema detection:}

The primary phase of calculation searches over all scales and image locations. It is employed proficiently by utilizing a difference-of-Gaussian function to recognize possible interest points that are invariant to scale and orientation.

\subsubsection{Key point localization:}

At every aspirant location, a comprehensive model is robust to decide location and scale. Key points are chosen based on procedures of their stability.

\subsubsection{Orientation assignment:}

One or more orientations are allocated to every key point position supported on local picture grade directions. All prospect operations are executed on image data that has been changed comparative to the dispensed orientation, scale, and location for every feature, thus given that invariance to these alterations.

\subsubsection{Key point descriptor:}

The confined image slopes are considered at the chosen scale in the province about each key point. These are changed into a illustration that permits for significant levels of restricted shape deformation and change in clarification.

For any objective there are many features available for medical images like hierarchical and spatial structure features, appealing points on the entity that can be mined to present a "characteristic" portrayal of the object. This portrayal can then be utilized when endeavoring to situate the object in a figure enclosing several other objects.

\subsection{Feature selection using automatic subjective optimality model}

After extracting the features using SIFT, selection of feature is done based on the respective pixel subjective points. Feature selection on the medical images is done in the previous work based on the automatic subjective-optimality model. Subjective optimality refers to the context of image analysis to be made i.e., tumor, non-tumor, and edema dependent feature sets. The feature point selection is realized by approximating for the subjective points like tumor section, non-tumor features within each feature points, the point of equivalence among the subjective parts between the crucial medical image and its consequent model constructed through the training stage. The automatic subjective optimality framework commences subjective optimal feature point selection the concept of subjective conditional probability, which illustrates the geometric allocation of a subjective point specified at the identified positions of a set of points.

\subsection{Multi-classifier framework using mutual information criterion}

After selecting the features, it is necessary to classify the images according to the class and type it belongs to. For classification of segmented image, here we proposed a multiclassifier framework. The multi-classifier is designed based on the mutual information coefficient of the selected features underwent for image segmentation procedures. The classification is done with set of rotation invariant features being selected on the lines of subjective-optimality and

\subsubsection{Estimation of Mutual information and multi- classifier framework}

different classifiers are organized d using different features sets trained in different data.

\subsubsection{Mutual information and its properties}

Mutual information (MI) is a crucial notion in all parts of applications and domains. It is a principle of common interdependence among arbitrary variables. Especially, specified two arbitrary variables $\mathrm{A}$ and $\mathrm{B}$, the mutual information $\mathrm{I}(\mathrm{A} ; \mathrm{B})$ is expressed as equation 1 ,

$$
\mathrm{I}(\mathrm{A}, \mathrm{B})=\mathrm{H}(\mathrm{A})+\mathrm{H}(\mathrm{B})-\mathrm{H}(\mathrm{A}, \mathrm{B})
$$

$H()$ is the entropy of a arbitrary variable and access the ambiguity connected with it. For an uninterrupted arbitrary variable $\mathrm{A}, \mathrm{H}(\mathrm{A})$ is expressed as equation 2 ,

$$
H(A)=-\int p(a) \log _{2} p(a) d a \ldots
$$

If $\mathrm{A}$ is a distinct, then $\mathrm{H}(\mathrm{A})$ is expressed as follows:

$$
H(A)=\sum p(A) \log _{2} p(A) \ldots(3)
$$

In both cases $\mathrm{p}(\mathrm{A})$ symbolizes the trivial possibility division of respective cases A. Based on the above equations, it is obvious why the entropy is regularly measured a measure of ambiguity.

The mutual information I (A: B) proceeds how much the insecurity of A is condensed if $B$ has been examined. It can be simply revealed that if $A$ and $B$ are usually self-sufficient, then $\mathrm{H}(\mathrm{A}, \mathrm{B})=\mathrm{H}(\mathrm{A})+\mathrm{H}(\mathrm{B})$. As a result their mutual information is zero, i.e., examining $B$ does not decrease the Improbability of A. If, conversely, $A=B$ then $I(A ; A)=H(A)$. Consequently, the entropy is also termed as self-information.

As mutual information creates no notions concerning the nature of the relationship among variables, it is pretty common and often observed as a simplification of the linear association co-efficient. If, conversely, A and B are Gaussian arbitrary variables, it has been revealed that their MI is a easy conversion of their linear correspondence co-efficient $\rho$.

$$
I(A, B)=-\frac{1}{2} \log \left(1-\rho^{2}\right)
$$

The notion of mutual information can be simply extended to comprise more than two arbitrary variables. Consistent with the chain rule, the joint mutual information (JMI) among a set of features $A_{1}, A_{2}, \ldots, A_{n}$ and the result $B$ i.e., diagnosis is

$$
I\left(A_{1}, A_{2}, \ldots, A_{n} ; B\right)=\sum_{i=1}^{N} I\left(A_{i} ; B \mid A_{i=1}, A_{i=2}, \ldots, A_{i}\right)
$$

JMI was commenced to portray how much the information presented by the feature vector $\mathrm{A}_{1}, \mathrm{~A}_{2}, \ldots, \mathrm{A}_{\mathrm{N}}$ reduces the improbability about the outcome $\mathrm{B}$. Given a huge set of features, it is projected that some of the features might be reliant on each other. Consequently, choosing features with their distinct MI concerning with the output can construct subsets that hold informative so far unnecessary features. JMI is a more suitable classification criterion as it can present an optimal subset that holds not only the most pertinent but also the least unnecessary features.

The inference of the mutual information criterion among a set of features and the given diagnosis is more difficult. For a set 
of $\mathrm{N}$ features, there are $2 \mathrm{~N}$ probable subsets. A comprehensive search needs that the MI is expected for every promising subset. For instance, with only 12 features, there are 4096 probable feature subsets to be measured. The feature subset that contains the highest MI with the output variable is the most favorable one. While the number of chosen features increases, exponentially other data models are essential for consistent MI estimation. The process of MI on segmented image is shown in figure 3 .

\begin{tabular}{|c|c|c|c|}
\hline $\mathrm{F} 1$ & f2 & & $\mathrm{f} 4$ \\
\hline F3 & f2 & f1 & f1 \\
\hline F4 & $\mathrm{f} 3$ & $f^{2}$ & f1 \\
\hline $\mathrm{F} 1$ & f4 & $f$ & $\mathrm{f} 3$ \\
\hline
\end{tabular}

Segmented image
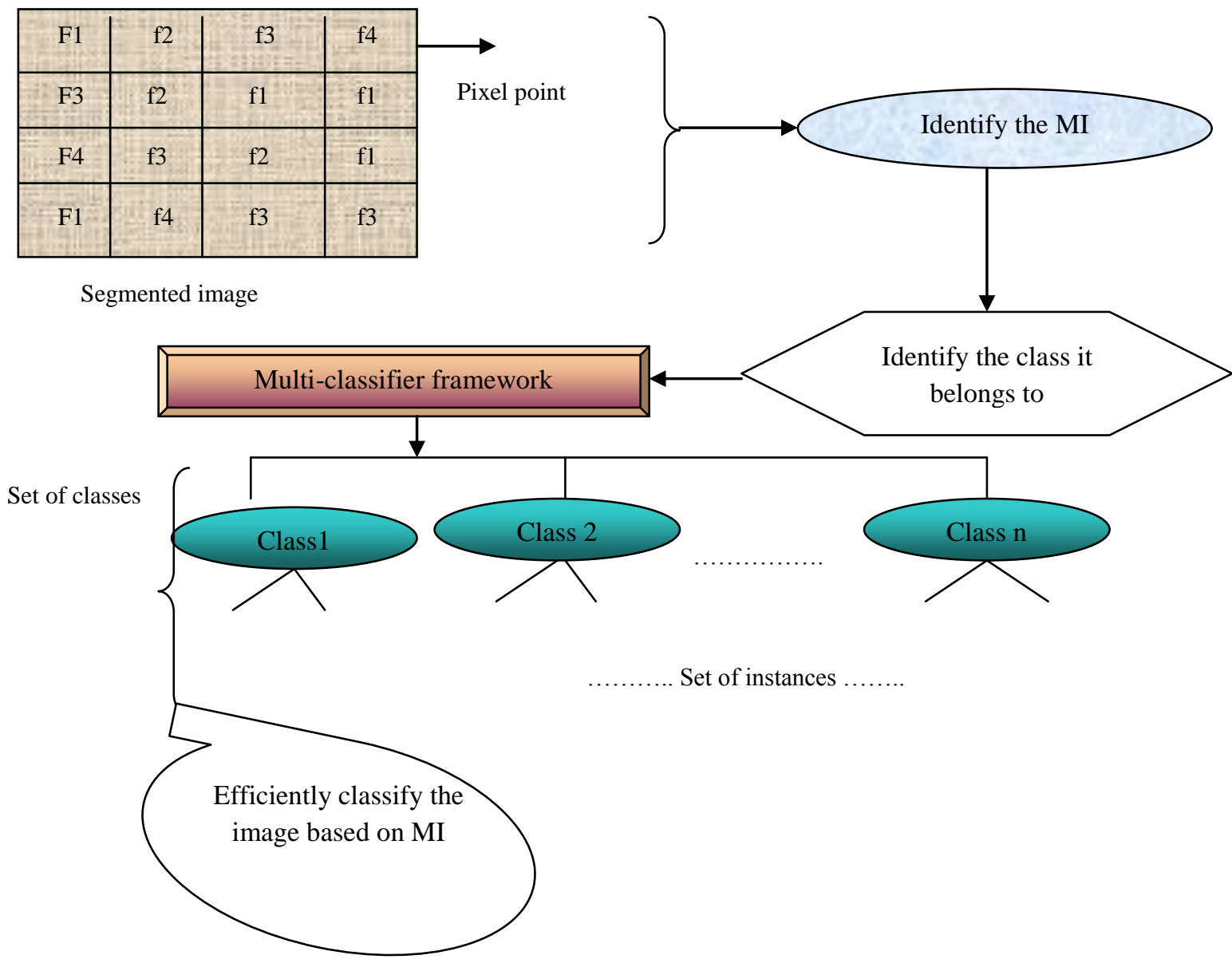

Figure 3: Process of MI on segmented image

After identification of the mutual information in the segmented image at each pixel point, the classification is done. A multiple classifier is presented for the medical images to classify the medical images based on the different number of classes like normal body cells, infected cells, and highly infected cells. Normally, an image has different number of classes which has several instances. To classify the image according to the class it belongs, mutual information criterion is utilized.

The multi-classifier is designed based on the mutual information coefficient of the selected features underwent for image segmentation procedures. The classification is done with set of rotation invariant features being selected on the lines of subjective-optimality and different classifiers are organized $\mathrm{d}$ using different features sets trained in different data. The method starts by choosing the distinct feature $A_{i}$ that has the maximum $\mathrm{MI}$ with the output variable $\mathrm{B}$ and expressed as,

$$
I\left(A_{j} ; B\right)-\beta \sum_{k} I\left(A_{k} ; A_{j}\right)
$$

Where $\mathrm{k}$ symbolizes pre-chosen features and $\mathrm{j}$ symbolizes the candidate features. Parameter $\mathrm{b}$ gets values among 0.5 and 1.0 and its best value is identified empirically. With the selected features and mutual information, the classification is done efficiently based on different number of classes and provides an efficient data retrieval process.

\section{EXPERIMENTAL EVALAUTION}

In this paper, the experimental simulation is conducted by using the medical image processing software package (MATLAB). An experimental performance is evaluated with benchmark data sets extracted from research repositories of 
both real and synthetic data sets. The medical image is given as input which includes features like size, shape, texture, spatial and hierarchical etc. The features are extracted by using SIFT described in our first work for medical image segmentation. Then the feature based image segmentation is achieved in our second work through the automatic subjective-optimality model. The schematic representation efficiently segments the given image based on different sets of selected features from the unsupervised learning model of extracted features. Subjective optimality refers to the context of image analysis to be made i.e., tumor, non-tumor, and edema dependent feature sets. In this work, a multiple classifier framework is done for the segmented medical images based on the normal body cells, infected cells, and highly infected cells. The classifier is designed based on the mutual information coefficient of the selected features underwent for image segmentation procedures. The classification is done with set of rotation invariant features being selected on the lines of subjective-optimality and different classifiers are organized d using different features sets trained in different data. During experimentation, a medical image is taken as input and processed with the schematic procedure for image segmentation and feature selection process. Then MI is identified for every pixel point of the segmented image. A multi-classifier framework is applied to evaluate the classification among the features obtained for the given medical image.

The performance of the proposed multi-classifier framework using mutual concept criterion [MFMCC] for medical image analysis is measured in terms of,

a. Mutual information coefficient of rich features

b. Time consumption

c. Efficiency.

\section{RESULTS AND DISCUSSION}

In this section, provide some experimental results to illustrate the effectiveness of the proposed multi-classifier framework using mutual concept criterion [MFMCC] for medical image analysis. The MFMCC scheme efficiently classified the medical images based on the normal body cells, infected cells, and highly infected cells. The classification is done with set of rotation invariant features being selected on the lines of subjective-optimality using different features sets trained in different data. To evaluate the efficiency of the proposed MFMCC, the results are compared with the existing local feature extraction method and with our previous works. The below table and graph describes the performance of the proposed multi-classifier framework using mutual concept criterion [MFMCC] for medical image analysis.
Table 1: No. of features vs. Mutual information

\begin{tabular}{|c|c|c|c|c|}
\hline \multirow{2}{*}{$\begin{array}{c}\text { No. of } \\
\text { features }\end{array}$} & \multicolumn{4}{|c|}{ Mutual information } \\
\cline { 2 - 5 } & $\begin{array}{c}\text { Proposed } \\
\text { MFMCC }\end{array}$ & FSASO & SIFT & $\begin{array}{c}\text { Existing } \\
\text { LFEM }\end{array}$ \\
\hline 3 & 0.09 & 0.04 & 0.05 & 0.02 \\
\hline 6 & 0.08 & 0.06 & 0.04 & 0.03 \\
\hline 9 & 0.12 & 0.07 & 0.06 & 0.05 \\
\hline 12 & 0.15 & 0.09 & 0.09 & 0.04 \\
\hline 15 & 0.19 & 0.11 & 0.07 & 0.03 \\
\hline
\end{tabular}

The above table 1 describes the mutual information coefficient for the set of features obtained in the segmented image. The mutual information co-efficient of the proposed multi-classifier framework using mutual concept criterion is compared with an existing local feature extraction method [LEFM] and with our previous works feature selection on segmented image using automatic subjective optimality model [FSASO] and Scale Invariant Feature Transform [SIFT].

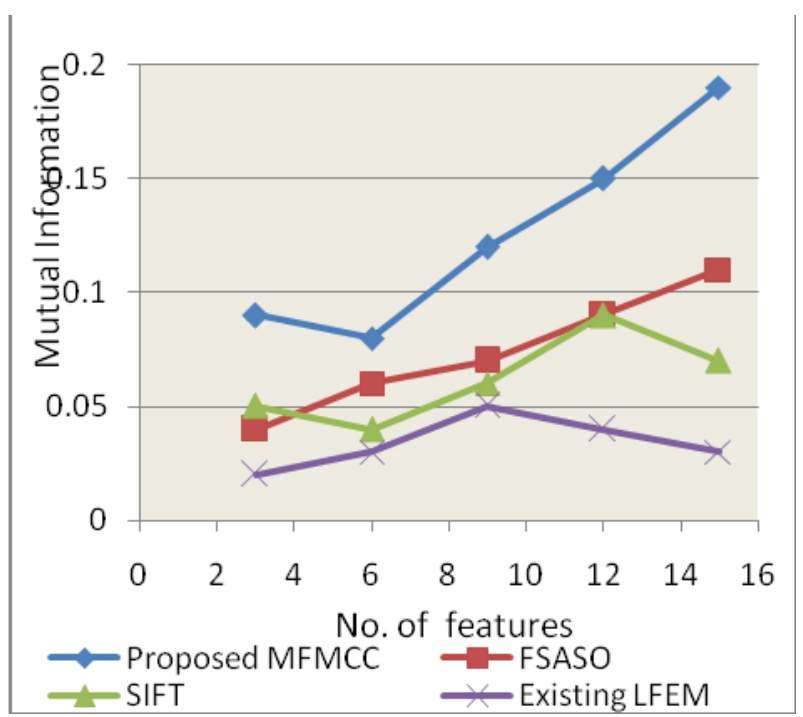

Figure 4: No. of features vs. Mutual information

Figure 4 describes the mutual information co-efficient for the set of features obtained in the segmented image. Mutual information is a determination of information content. By itself, it could be employed to assess the information substance of a specified feature or set of features and the preferred output i.e., accurate diagnosis. Individual features or subsets of features with small content information can be disqualified from the specified scheme. Exploiting a principle to eradicate inappropriate or unnecessary features is by no means a distinctive concept. By obtaining the mutual information, we can easily classify the medical images based on the normal body cells, infected cells, and highly infected cells. The mutual information of the segmented image is clearly obtained only in the proposed MFMCC. Compared to the existing local feature extraction method [LEFM] and our 
previous works feature selection on segmented image using automatic subjective optimality model [FSASO], Scale Invariant Feature Transform [SIFT], the proposed MFMCC provides an efficient mutual information co-efficient for rich featured for medical image analysis. The variance in the mutual information co-efficient is 50-60 \% high in the proposed MFMCC.

Table 2: No. of features vs. Time consumption

\begin{tabular}{|c|c|c|c|c|}
\hline \multirow{2}{*}{$\begin{array}{c}\text { No. of } \\
\text { features }\end{array}$} & \multicolumn{4}{|c|}{ Time consumption (seconds) } \\
\cline { 2 - 5 } & $\begin{array}{c}\text { Proposed } \\
\text { MFMCC }\end{array}$ & FSASO & SIFT & $\begin{array}{c}\text { Existing } \\
\text { LFEM }\end{array}$ \\
\hline 3 & 12.2 & 15.4 & 17.2 & 20.6 \\
\hline 6 & 12.5 & 16.7 & 19.6 & 21.8 \\
\hline 9 & 14.6 & 18.4 & 21.2 & 23.6 \\
\hline 12 & 15.3 & 20.4 & 23.8 & 24.8 \\
\hline 15 & 16 & 21.4 & 24.3 & 26.7 \\
\hline
\end{tabular}

The above table 2 describes the consumption of time needed to perform the classification of rich features based on the number of features available in the segmented image. The time consumption of the proposed multi-classifier framework using mutual concept criterion is compared with an existing local feature extraction method [LEFM] and with our previous works feature selection on segmented image using automatic subjective optimality model [FSASO] and Scale Invariant Feature Transform [SIFT].

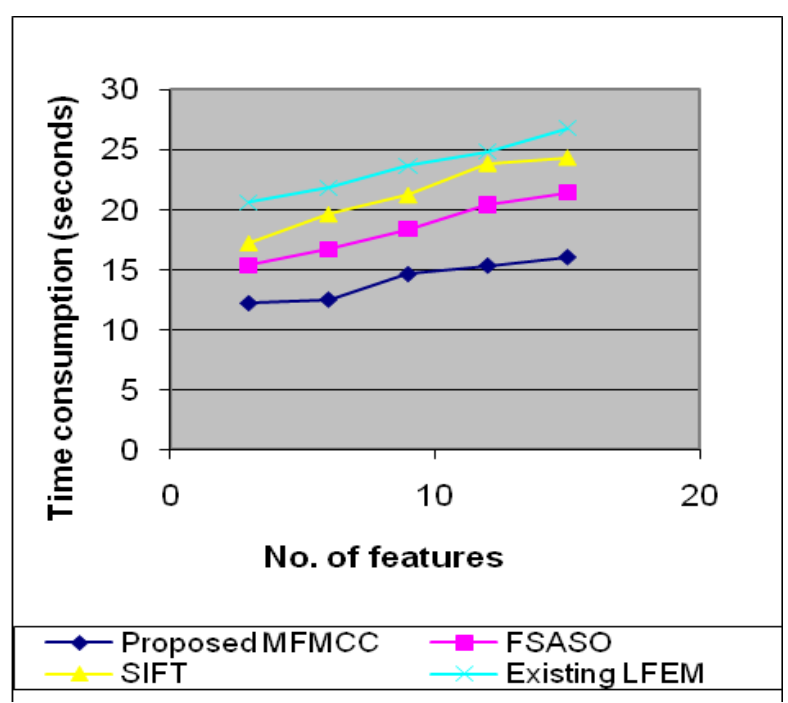

Figure 5: No. of features vs. Time consumption

Figure 5 describes the consumption of time needed to perform the classification of rich features based on the number of features available in the segmented image. Since the proposed MFMCC followed the process of mutual information co-efficient for classification. The mutual information concept efficiently obtained the information at each pixel point in the segmented image. So, it is easy to classify the image based on the information obtained. The classification is done with set of rotation invariant features being selected on the lines of subjective-optimality and different classifiers are organized using different features sets trained in different data. The time consumption is measured in terms of seconds. Compared to the existing local feature extraction method [LEFM] and our previous works feature selection on segmented image using automatic subjective optimality model [FSASO], Scale Invariant Feature Transform [SIFT], the proposed MFMCC provides an efficient classification of medical images in a less interval of time. The variance in the consumption of time is $60-70 \%$ low in the proposed MFMCC.

Table 3: No. of pixels vs. Efficiency

\begin{tabular}{|c|c|c|c|c|}
\hline \multirow{2}{*}{$\begin{array}{c}\text { No. of } \\
\text { pixels in } \\
\text { size }\end{array}$} & \multicolumn{4}{|c|}{ Efficiency (\%) } \\
\cline { 2 - 5 } & $\begin{array}{c}\text { Proposed } \\
\text { MFMCC }\end{array}$ & FSASO & SIFT & $\begin{array}{c}\text { Existing } \\
\text { LFEM }\end{array}$ \\
\hline 100 & 64 & 54 & 50 & 45 \\
\hline 200 & 70 & 60 & 55 & 50 \\
\hline 300 & 75 & 63 & 59 & 54 \\
\hline 400 & 80 & 68 & 64 & 58 \\
\hline 500 & 84 & 73 & 68 & 64 \\
\hline
\end{tabular}

The table 3 describes the efficiency of classification of rich features based on the number of pixels in the given medical image. The efficiency of the proposed multi-classifier framework using mutual concept criterion is compared with an existing local feature extraction method [LEFM] and with our previous works feature selection on segmented image using automatic subjective optimality model [FSASO] and Scale Invariant Feature Transform [SIFT]

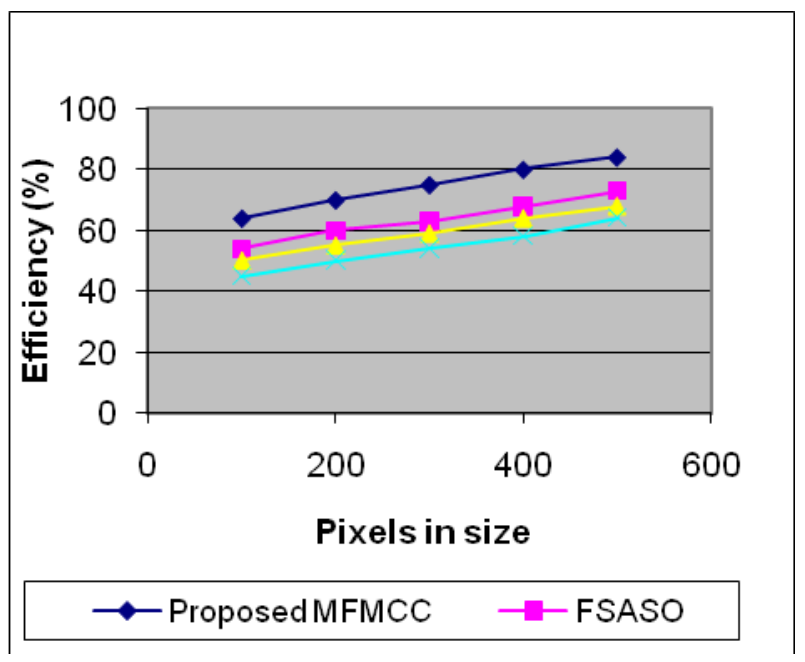

Figure 6 No. of pixels vs. Efficiency 
Figure 6 describes the efficiency of classification of rich features based on the number of pixels in the given medical image. In the proposed MFMCC, the classification of segmented image is done reliably with the mutual information co-efficient concept of the selected features underwent for image segmentation procedures. SO compared to the other works, the proposed multi-classifier framework using mutual concept criterion provides a reliable medical image analysis in terms of classification, segmentation and time consumption.

Finally, it is being observed that the proposed MFMCC classified the medical images based on the normal body cells, infected cells, and highly infected cells. The classifier is designed reliably based on the mutual information coefficient of the selected features underwent for image segmentation procedures. The classification is done with set of rotation invariant features being selected on the lines of subjectiveoptimality and different classifiers are organized using different features sets trained in different data.

\section{CONCLUSION}

Multiple classifier frameworks for the medical images to achieve the rich feature image classification based on different number of classes like normal body cells, infected cells, and highly infected cells. The classifier is designed based on the mutual information coefficient of the selected features underwent for image segmentation procedures. The classification is done reliably with set of rotation invariant features being selected on the lines of subjective-optimality and different classifiers are organized using different features sets trained in different data. The unsupervised learning model is used to extract features from the medical images using spatial and hierarchical structures based on scale invariant feature transformation. This would enrich the features extracted from the medical image for segmentation compared to the existing method features of intensity, FD and shape model. To be clear, this work showed that the mutual information is a promising classification criterion for medical image analysis. An extensive evaluation is carried out to evaluate the performance of the proposed multi-classifier framework using mutual concept criterion. An evaluation concluded that the proposed MFMCC is better in terms of mutual information, time consumption and its efficiency compared to the existing local feature extraction method [LEFM] and with our previous works feature selection on segmented image using automatic subjective optimality model [FSASO] and Scale Invariant Feature Transform [SIFT].

\section{REFERENCES}

[1] Dame, A. et. Al., "Second-Order Optimization of Mutual Information for Real-Time Image Registration", IEEE Transactions on Image Processing, 2012

[2] Loeckx, D. et. Al., "Nonrigid Image Registration Using Conditional Mutual Information", IEEE Transactions on Medical Imaging, 2010

[3] Ashraf, A. et. Al., "A Multichannel Markov Random Field Framework for Tumor Segmentation with an Application to Classification of Gene Expression-based Breast Cancer Recurrence Risk.", IEEE Transactions on Medical Imaging, 2012

[4] Kerroum, M.A.et. Al., "Textural feature selection by mutual information for multispectral image classification", International Conference on Multimedia Computing and Systems (ICMCS) 2009.

[5] Hossain, M.A. et. Al., "Unsupervised feature extraction based on a mutual information measure for hyperspectral image classification”, IEEE International Geoscience and Remote Sensing Symposium (IGARSS), 2011

[6] $\mathrm{Hu}, \mathrm{M}$. et. Al., "Maximisation of mutual information for gait-based soft biometric classification using gabor features", Biometrics, IET, 2012

[7] Carmona, P.L. et. Al., "Band selection in spectral imaging for classification and regression tasks using information theoretic measures", $10^{\text {th }}$ Euro-American Workshop on Information Optics (WIO), 2011

[8] Qiang Wang et. Al., "Shared chain mutual information measure for image fusion", IEEE International Conference on Automation and Logistics, 2008. ICAL 2008.

[9] Michel, V. et. Al., "Mutual information-based feature selection enhances FMRI brain activity classification", 5th IEEE International Symposium on Biomedical Imaging, 2008.

[10] El Maia, H. et. Al., "Hybridization of color information with texture by using mutual information for the classification of satellite images", International Conference on Multimedia Computing and Systems, 2009. ICMCS '09.

[11] H.P. Ng et. Al., "Medical Image Segmentation using Kmeans Clustering and Improved Watershed Algorithm", IEEE Southwest Symposium on Image Analysis and Interpretation, 2006

[12] Gurbinder Kaur et. Al., "Intensity based Image Segmentation using Wavelet Analysis and Clustering Techniques", Indian Journal of Computer Science and Engineering (IJCSE) ISSN: 0976-5166 Vol. 2 No. 3 JunJul 2011 